\title{
Multi-gas assessment of the Kyoto Protocol
}

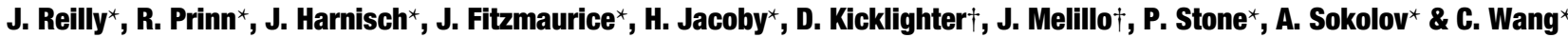

* Joint Program on the Science and Policy of Global Change, Massachusetts Institute of Technology, 77 Masachusetts Avenue, Building E40, Cambridge, Massachusetts 02139, USA

$\dagger$ The Ecosystems Center, Marine Biological Laboratory, Woods Hole, Massachusetts 02543, USA

The Kyoto Protocol allows reductions in emissions of several 'greenhouse' gases to be credited against a $\mathrm{CO}_{2}$-equivalent emissions limit, calculated using 'global warming potential' indices for each gas. Using an integrated global-systems model, it is shown that a multi-gas control strategy could greatly reduce the costs of fulfilling the Kyoto Protocol compared with a $\mathrm{CO}_{2}$-only strategy. Extending the Kyoto Protocol to 2100 without more severe emissions reductions shows little difference between the two strategies in climate and ecosystem effects. Under a more stringent emissions policy, the use of global warming potentials as applied in the Kyoto Protocol leads to considerably more mitigation of climate change for multi-gas strategies than for the-supposedly equivalent- $\mathrm{CO}_{2}$-only control, thus emphasizing the limits of global warming potentials as a tool for political decisions.

Many trace atmospheric constituents affect the radiative budget of the atmosphere. The Kyoto Protocol includes carbon dioxide $\left(\mathrm{CO}_{2}\right)$, methane $\left(\mathrm{CH}_{4}\right)$, nitrous oxide $\left(\mathrm{N}_{2} \mathrm{O}\right)$, perfluorocarbons (PFCs), hydrofluorocarbons (HFCs), and sulphur hexafluoride (SF S $_{\text {) }}$ http://www.unfccc.de/resource/docs/convkp/kpeng.pdf). Some studies suggest that potential carbon sinks ${ }^{1,2}$ and opportunities to limit emissions of other greenhouse gases ${ }^{3-5}$ may reduce the cost of control. These also point to the risks of failing to control gases with very long lifetimes ${ }^{3,6,7}$. Few studies have yet considered an integrated evaluation of the costs of multi-gas control strategies, or the implications of reductions in different mixes of greenhouse gases on atmospheric composition, climate and ecosystems $\mathrm{s}^{8-11}$.

Using the MIT Integrated Global System Model $^{12}$ (IGSM) as recently modified ${ }^{13}$ we examine multi-gas control as envisioned by the Kyoto Protocol, exploring the costs of emissions reduction and the consequences for the atmosphere, climate and ecosystems. The basic components of the IGSM are an emissions prediction and policy analysis (EPPA) model $^{14}$, a natural emissions model, a coupled atmospheric chemistry and climate model ${ }^{15,16}$, and a terrestrial ecosystems model ${ }^{17}$. We stop short of quantifying damages in monetary terms. Our ecosystems results illustrate trade-offs that result from different control strategies. We find that inclusion of sinks and abatement opportunities for gases other than $\mathrm{CO}_{2}$ could reduce the cost of meeting the Kyoto agreement by $60 \%$. Assuming the protocol is extended unchanged to 2100 , we find little difference in climate and ecosystem effects between 2010 and 2100 for a strategy that achieves the required reduction with a multi-gas strategy as compared to a $\mathrm{CO}_{2}$-only strategy. Under a more aggressive policy, increasing the reductions in Annex B countries (Eastern and Western Europe, Russian Federation, Australia, New Zealand, Japan, Canada and the United States) and extending reductions to the rest of the world after 2010, significant differences in effects develop between the two strategies. This latter result indicates that 100 -year global warming potentials (GWPs), as currently estimated, fail to capture important time horizon and climate-chemistry interactive effects, and this failure may be significant for policy.

\section{Policy cases}

The Kyoto Protocol establishes maximum allowable emissions of greenhouse gases aggregated using 100-year GWPs for the period 2008 to 2012 for each party in Annex B of the agreement, stated as a percentage of base year emissions. Global warming potentials are indices that supposedly establish equivalency in terms of climate effects for purposes of comparing reductions in emissions of different greenhouse gases to assess compliance with the Kyoto agreement ${ }^{18}$. The motivation is that such gases have very different lifetimes and specific radiative forcing and thus are not at all comparable on a tonne-for-tonne basis. The Kyoto Protocol also allows credit for carbon sinks resulting from direct, human-induced afforestation and reforestation measures occurring after 1990. The Protocol rules out credit for indirect sink enhancement from increased growth of plants due to increased atmospheric $\mathrm{CO}_{2}$ itself or from deposition of nitrogen emitted from industrial processes. Thus, although the role of terrestrial ecosystems in balancing the carbon budget is important in understanding the carbon cycle ${ }^{19}$ the magnitude of a natural land sink is irrelevant to Kyoto accounting. Inclusion of agricultural soils and a broader set of afforestation activities are under discussion.

Global warming potentials hide complex feedbacks among ecosystems, the atmosphere, and the oceans, and omit responses that are nonlinear with changing levels and rates of increase of different gases. They also ignore chemical interactions among the greenhouse gases and other gases, including processes of formation and destruction that depend on climate itself ${ }^{15}$. Patterns of forecast climate change may differ under alternative combinations of gas controls because spatial and transient forcing responses can be heterogeneous ${ }^{20}$. Also, GWPs depend on the choice of time horizon for integration of the effects of radiative forcing. For example, defined relative to $\mathrm{CO}_{2}\left(\mathrm{CO}_{2}=1.0\right)$, the shorter-lived $\mathrm{CH}_{4}$ has a higher GWP with a shorter time horizon (21 under a 100-year horizon, 56 under a 20 -year horizon $)^{18}$. An alternative to using a finite time horizon is to formulate GWPs as a solution to an infinite horizon economic problem with a discount rate to provide an economic weighting of gases over time. Global warming potentials would then depend on the actual effects of climate change and greenhouse-gas accumulation on the economy, human wellbeing, and ecosystems. Such a calculation requires effects to be estimated in a comparable measure, usually in monetary (for example, US dollar) terms ${ }^{21-23}$. Carbon dioxide also has direct effects on plant growth that should be included in damage estimates. Their inclusion would further affect GWP calculations ${ }^{22}$. There are significant limitations on existing efforts to value damages in monetary terms ${ }^{24}$, however, and controversy about the use of discounting in climate change policy analysis ${ }^{25}$.

In addition to a future reference case with no emission controls, 
we develop three basic policy cases to test the economic importance of including non- $\mathrm{CO}_{2}$ gases in the Kyoto Protocol.

Case 1. Fossil $\mathrm{CO}_{2}$ target and control. This case is representative of much previous work on the costs of limiting the greenhouse effect. It includes only $\mathrm{CO}_{2}$ in determining the allowable emissions, unlike the requirements in the Kyoto Protocol that consider multiple gases. Case 2. Multi-gas target with control on $\mathrm{CO}_{2}$ emissions only. This case is constructed with the multi-gas target (expressed as carbon equivalents using GWPs), as described in the Kyoto Protocol, but only carbon emissions from fossil fuels are controlled.

Case 3. Multi-gas target and controls. The multi-gas Kyoto target applies in this case, and parties seek the least cost control across all gases and carbon sinks.

For purposes of analysis of climate and ecosystem effects over the longer term we extend policy cases 2 and 3 under two sets of assumptions. In one we assume that the Kyoto commitment remains unchanged to the end of 2100 (extended cases 2 and 3 ). We also define much more stringent versions of cases 2 and 3, denoted cases $2^{\prime}$ and $3^{\prime}$ respectively. In these latter cases Annex B reduces carbon-equivalent emissions by an additional $5 \%$ in each succeeding 15 -year period, to $35 \%$ below 1990 levels by 2100 . We assume that non-Annex B regions join in 2025, adopting a target of $5 \%$ below their 2010 emissions. They then follow Annex B by tightening the constraint by $5 \%$ every 15 years, reaching $30 \%$ below 2010 emissions in 2100.

We assume in case 3 that non- $\mathrm{CO}_{2}$ gas abatement beyond 2010 is the same percentage below base levels as calculated for 2010, based on our economic assessment for 2010 and assuming also that the contribution from sinks remains unchanged through 2100. Case 2 maintains the same carbon-equivalent emissions with $\mathrm{CO}_{2}$-only controls, as calculated using 100-year GWPs. For case $3^{\prime}$ we assume that reductions beyond 2010 for all gases are proportional to the reduction commitment, and case $2^{\prime}$ again keeps the same carbonequivalent path, with control on $\mathrm{CO}_{2}$ only. The purpose of the extended cases is to examine whether the choice of gases to control makes a difference to the climate system. Cases $2^{\prime}$ and $3^{\prime}$ in particular differ significantly in emissions of both $\mathrm{CO}_{2}$ and other gases. As a result, they provide a good test of the climate and ecosystem implications of using the 100-year GWPs with different strategies for implementing the agreement.

Figure 1a shows that the difference between anthropogenic $\mathrm{CO}_{2}$ (plus $\mathrm{CO}$, which converts to $\mathrm{CO}_{2}$ ) emissions and total natural $\mathrm{CO}_{2}$ sinks (predicted for the ocean and prescribed for the land ${ }^{12}$ ) grows with time in the reference case and cases 2 and 3 but stabilizes or falls in cases $2^{\prime}$ and $3^{\prime}$. Reference emissions of carbon reach 17.4 gigatonnes $(\mathrm{Gt})$ by 2100 , lower than the previous EPPA reference of $20.6 \mathrm{Gt}$ (ref. 12). When combined with other greenhouse gas emissions using 100-year GWPs, total emissions reach about $25 \mathrm{Gt}$ C equiv. (gigatonnes of carbon equivalent) by 2100 (Fig. 1b). Under cases 2 and 3, combined annual emissions are reduced by about $7 \mathrm{Gt} C$ equiv. in 2100, whereas emissions are reduced by about 17 Gt $C$ equiv. in cases $2^{\prime}$ and $3^{\prime}$.

\section{Economic assessment}

Our economic analysis is limited to provisions of the Kyoto Protocol directed at Annex B countries, and focuses on the year 2010 as indicative of the annual cost in the Kyoto commitment period of 2008-12. Our approach uses marginal abatement curves (MACs) that allow the options available for abating emissions to be ordered from lowest cost to highest cost. The significance of this ordering is that an economically efficient policy would choose the lowest-cost option first, moving to higher-cost options as more abatement was required. Figure 2 shows MACs for $\mathrm{CO}_{2}$ and all greenhouse gases for the USA, illustrating our approach and how it relates to the policy cases we examine. Marginal abatement curves for individual gases and sinks are shown in the inset. For the USA, the required reduction under case 1 (RR1) of $571 \mathrm{MtC}$ equiv. (megatonnes of carbon equivalent) results in a price (P1) of $\$ 187$ per tonne of carbon equivalent ( $\mathrm{t} C$ equiv.). With a multi-gas target the required reduction ( $\mathrm{RR} 2,3$ ) rises to $650 \mathrm{Mt} \mathrm{C}$ equiv. If this target must be achieved with controls on only $\mathrm{CO}_{2}$ emissions, then the price rises to $\$ 229$ per t C equiv., but taking advantage of abatement options for all gases and sinks reduces the price to $\$ 150$ per t C equiv.

The base year (1990) emissions, 2010 reference, and allowable emissions under the policy cases representing the Kyoto Protocol in our analysis (in carbon equivalent using 100-year GWPs) are presented in Table 1, along with the required reductions. Reference emissions are projected in our analysis to increase substantially by 2010 in all regions except the former Soviet Union (FSU). The increases from the base year range from $29 \%$ in the European Union (EEC) to $46 \%$ in Eastern Europe (EET). Aggregate emissions in the FSU are projected to be $7.5 \%$ less in 2010 than in 1990, most of this reduction having already occurred before 1998. The allowable emissions for the FSU exceed our reference emissions in 2010, giving the FSU 'hot air' that can be traded or banked against future increases in emissions. Including the non-carbon gases in calculating our reference emissions reduces this 'hot air' by nearly $60 \%$, reflecting the fact that non- $\mathrm{CO}_{2}$ gases in the $\mathrm{FSU}$ are projected to grow in our reference scenario. This reduction is mainly due to accounting for methane emissions. However, our model includes no direct measurements of methane emissions or abatement potential in the FSU region. 'Hot air' calculations are, in general, highly
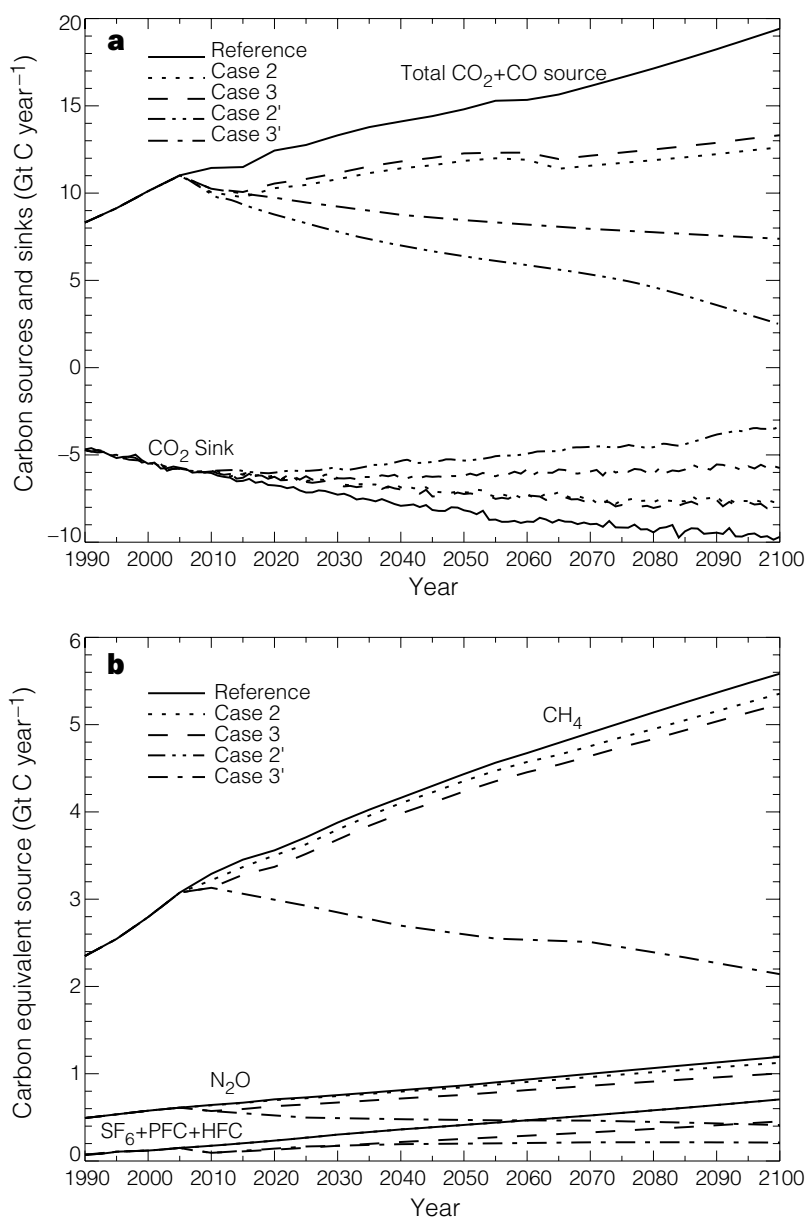

Figure 1 Future greenhouse gases emissions. Panel a shows total $\mathrm{CO}_{2}$ and $\mathrm{CO}$ (which converts to $\mathrm{CO}_{2}$ ) sources (positive) and total $\mathrm{CO}_{2}$ sinks (negative) as references, and cases $2,2^{\prime}, 3$ and $3^{\prime}$. Panel $\mathbf{b}$ shows non- $\mathrm{CO}_{2}$ sources expressed as equivalent amounts of $\mathrm{CO}_{2}$ emissions using global warming potentials with 100 -year horizons. The case 2 ' plot lies on top of the reference plot in panel $\mathbf{b}$. 
uncertain. Another recent forecast of carbon emissions for the FSU implies that 'hot air' in 2010 will range up to $388 \mathrm{Mt}$ (ref. 26).

We computed MACs for $\mathrm{CO}_{2}$ reduction in 2010 using the EPPA model. Those for sinks and gases other than $\mathrm{CO}_{2}$ were developed from detailed evaluations of economic reduction potential drawn from published literature, recent studies, and cost estimates of industry experts. Parameter estimates for the curves are given in Fig. 1. Marginal abatement curves for sinks were developed based on a US study of forest carbon sequestration on agricultural land ${ }^{1}$. Quantities of sequestration for different regions available in 2010 were based on tree growth potential on currently idle or underused land, with regional MACs shifted horizontally to fit these regional quantities $^{27}$. We phased in the tree-planting programme as if it started in the year 2000 .

The potential for $\mathrm{CH}_{4}$ reduction was based on an assessment of the costs of recovery from landfills, livestock waste, coal seams, and oil and gas production conducted for the USA ${ }^{28,29}$. Other EPPA regions were assumed to face similar costs for comparable shares of abatement of $\mathrm{CH}_{4}$ emissions from the reference level. No $\mathrm{CH}_{4}$ abatement is included for ruminant animals or rice production. Existing economic studies show the marginal costs to be very high for these latter sources ${ }^{1}$, although these studies do not consider technological options that have been suggested more recently ${ }^{30}$. The reduction potential for $\mathrm{N}_{2} \mathrm{O}$ from fertilized soils was based on econometric studies of the price response of fertilizer demand ${ }^{31,32}$. Additional reduction potential is assumed to arise from switching

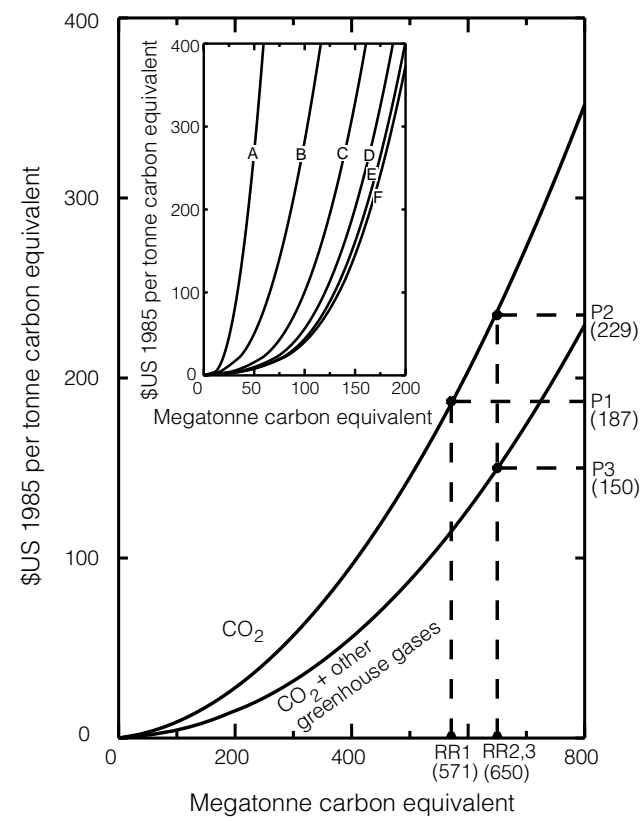

Figure 2 Marginal abatement curves (MACs). Calculated for $\mathrm{CO}_{2}$ in the USA and total greenhouse gases. RR1, RR2,3 and P1, P2, P3 are the required reductions and prices under policy cases 1, 2 and 3. Inset provides sequentially summed MACs for other greenhouse gases in the USA in carbon equivalent units using 100-year global warming potentials. A: $\mathrm{CH}_{4} ; \mathrm{B}: \mathrm{CH}_{4}+$ carbon sink; $\mathrm{C}: \mathrm{CH}_{4}+$ sink $+\mathrm{N}_{2} \mathrm{O} ; \mathrm{D}: \mathrm{CH}_{4}+$ sink + $\mathrm{N}_{2} \mathrm{O}+\mathrm{HFCS} ; \mathrm{E}^{-} \mathrm{CH}_{4}+\operatorname{sink}+\mathrm{N}_{2} \mathrm{O}+\mathrm{HFCS}+\mathrm{SF}_{6} ; \mathrm{F}: \mathrm{CH}_{4}+\operatorname{sink}+\mathrm{N}_{2} \mathrm{O}+\mathrm{HFCs}+$ $\mathrm{SF}_{6}+\mathrm{PFCs}$. Marginal abatement curves for the forest sink and $\mathrm{CH}_{4}$ are quadratic polynomials $\left(P=a+b Q+c Q^{2}\right)$ where $Q$ is the reduction in MtC equiv. and $P$ is the price in 1985 US dollars. For sinks: $a=33.5, b=-3.59, c=0.177$; for $\mathrm{CH}_{4}$ : $a=12.15, b=-2.42, c=0.15$. Parameters for other regions are available from the authors upon request. Other MACs are exponential functions $(P=a \exp (b S))$ where $S$ is the share of reference emissions reduced. For $\mathrm{N}_{2} \mathrm{O}: a=0.580, b=0.177$; for PFCs: $a=6.932, b=0.036$; for HFCs: $a=1.189, b=0.060$; for SF$: a=0.795$,

$b=0.065$, identical across regions. $\mathrm{P} 3$ given here is without "free" reductions of $\mathrm{CH}_{4}$ and $\mathrm{N}_{2} \mathrm{O}$ generated by the carbon constraint. between different types of synthetic fertilizers. Abatement from adipic and nitric acid production was based on industry estimates.

Estimates of the reduction potential and cost for other gases were based on information from the published literature and industry experts. Two main sources of HFCs are $\mathrm{CHF}_{3}$ as a by-product of hydrochlorofluorocarbon (HCFC) manufacture ${ }^{33}$ and $\mathrm{CH}_{2} \mathrm{FCF}_{3}$ from mobile air conditioning ${ }^{34}$. Their use in stationary cooling, foam blowing, and solvents is likely to grow rapidly. We considered recovery and thermal oxidation of by-products of HCFC production and replacement of HFCs with $\mathrm{CO}_{2}$ in mobile air conditioners (J. Wertenbach and R. Caesar, personal communication), and recovery and replacement options for the remaining sources. Perfluorocarbons such as $\mathrm{CF}_{4}$ are emitted during production of primary aluminium ${ }^{5}$ and semiconductors, and through their use as a replacement for ozone-depleting substances phased out under the Montreal Protocol. We estimated reduction rates and costs for the aluminium industry ${ }^{5}$. Also, costs of recovery from gas streams and subsequent combustion, and costs of substitute chemicals that were assumed to be available to the semiconductor sector and to other miscellaneous sectors were estimated. Expert estimates were the basis for the costs of removal of PFCs from waste gases in the semiconductor industry and for the costs of substitute chemicals in solvent applications.

Sulphur hexafluoride is emitted from magnesium and semiconductor production, the manufacture and use of electrical switchgear $^{4}$, and a broad category of other applications ${ }^{35}$. For losses in switchgear manufacturing we account for the fact that recycling of $\mathrm{SF}_{6}$ is economical at current prices, and assume rates of post-installation control at higher prices. Similarly, it is assumed that, at increased prices, magnesium producers will be able to cut specific emissions to values already achieved by Norwegian manufacturers ${ }^{35}$.

The MACs for 2010 then allow study of the policy cases presented in the previous section. Case 1 allows comparison with previous studies that have considered only $\mathrm{CO}_{2}$. Compared with case 3 it shows an overestimate of the carbon price ranging from about $8 \%$ in the EET ( $\$ 11)$ to $153 \%$ in the remainder of the OECD (OOE) (\$158); costs throughout are in 1985 \$US. Much of the difference comes from variation in the carbon sink potential among the regions. For example, there is relatively small sink potential in the EET and Japan and a very large sink potential relative to carbon emissions in the OOE. In terms of total cost, we find that, for Annex $\mathrm{B}$ as a whole, the error from leaving out other greenhouse gases is about a $\$ 27$ billion per year overestimate of the annual cost in 2010 (Table 2). The OOE, USA and EEC have substantially lower costs under case 3 than case 1 . Notably, however, case 3 achieves a greater reduction in greenhouse gases $(1.55$ versus $1.34 \mathrm{Gt} C$ equiv. per year) than case 1 .

Cases 2 and 3 were designed to be comparable in terms of carbonequivalent emissions abated; differences in costs thus reflect different options for implementing the agreement. We find that the carbon-equivalent price is over $\$ 100$ per tonne higher for the USA, EEC, OEE and Japan in case 2 than in case 3 . In terms of total annual cost the difference between cases 2 and 3 is striking. To achieve the 1.55 Gt C equiv. reduction only through fossil energy carbon emissions would cost nearly $\$ 62$ billion per year more than using a multi-gas strategy with sink enhancement, increasing the cost of the agreement by over $60 \%$. The EET is the only region with higher costs when other greenhouse gases and sinks are included. This happens because of their limited potential for sinks and the fact that emissions for non- $\mathrm{CO}_{2}$ greenhouse gases are growing rapidly in the reference case. The multi-gas strategy in the Kyoto Protocol thus creates an opportunity for lower abatement costs, but introduces a risk of higher costs if reductions in these other gases are not made.

Some studies show a potential even larger than we have estimated for reducing non- $\mathrm{CO}_{2}$ greenhouse gases ${ }^{36}$. Leakage rates of $\mathrm{CH}_{4}$ from gas and coal mine operations in countries of the FSU are 


\section{Table 1 Emissions scenarios}

\begin{tabular}{|c|c|c|c|c|c|c|}
\hline Gas & USA & EEC & OOE & EET & JPN & FSU \\
\hline \multicolumn{7}{|c|}{ Base year (1990) emissions; (2010 Reference forecast) ${ }^{*}$} \\
\hline $\mathrm{CO}_{2}$ & $1,362(1,838)$ & $822(1,064)$ & $318(472)$ & $266(395)$ & $298(424)$ & $891(763)$ \\
\hline $\mathrm{CH}_{4}$ & $170(184)$ & $129(143)$ & $69.9(84.8)$ & $64.5(82.1)$ & $9.0(10.9)$ & $155(209)$ \\
\hline $\mathrm{N}_{2} \mathrm{O}$ & $92.3(121)$ & $71.7(92.3)$ & $23.4(31.9)$ & $8.9(10.6)$ & $9.3(13.7)$ & $11.3(14.2)$ \\
\hline $\mathrm{SF}_{6}$ & $10.5(12.7)$ & $6.2(7.7)$ & $3.3(4.2)$ & $1.0(1.6)$ & $3.5(4.9)$ & $3.2(3.5)$ \\
\hline HFC & $12.3(27.6)$ & $7.6(17.7)$ & $2.5(4.2)$ & $0.5(1.7)$ & $4.6(11.6)$ & $1.6(4.6)$ \\
\hline PFC & $7.4(5.0)$ & $4.1(2.8)$ & $7.2(5.1)$ & $1.1(0.9)$ & $1.3(0.3)$ & $6.2(5.3)$ \\
\hline Total & $1,654(2,188)$ & $1,042(1,327)$ & $425(604)$ & $342(492)$ & $326(466)$ & $1,068(1,000)$ \\
\hline Kyoto reduction (below 1990) \% & -7.0 & -8.0 & -5.5 & -7.0 & -6.0 & -0.2 \\
\hline \multicolumn{7}{|c|}{ Allowed anthropogenic emissions; (required reductions)* } \\
\hline Case 1 & $1,267(571)$ & 757 (308) & $301(171)$ & $248(147)$ & $280(144)$ & $889(-126)$ \\
\hline Cases 2 and 3 & $1,539(650)$ & $958(369)$ & $401(203)$ & $318(174)$ & $306(160)$ & $1,066(-66)$ \\
\hline
\end{tabular}

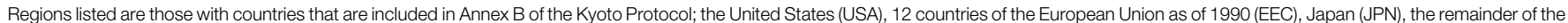

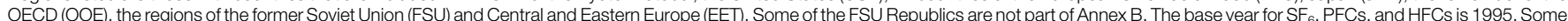

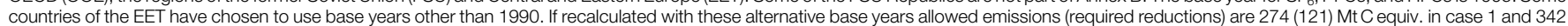
(150) Mt C equiv. in cases 2 and 3.

reported to be high, and it may well be economic to reduce these leakage rates even without climate policy. If so, these reductions in the FSU could be less expensive than represented in our analysis. The cost savings could thus be even larger and the scope for trade in emissions permits would be expanded. 'Hot air' would also then increase substantially beyond our estimate. Further refinement of region-specific costs is needed in order to understand the implications for trading opportunities better. Although the above caveats suggest lower marginal abatement costs, realization of the potential for reductions in other greenhouse gases and increased sinks requires that policies and economic incentives be put in place to bring these reductions about.

Our analysis assumes policies that achieve reductions at least cost. We have included 'no-cost' reduction options in our reference
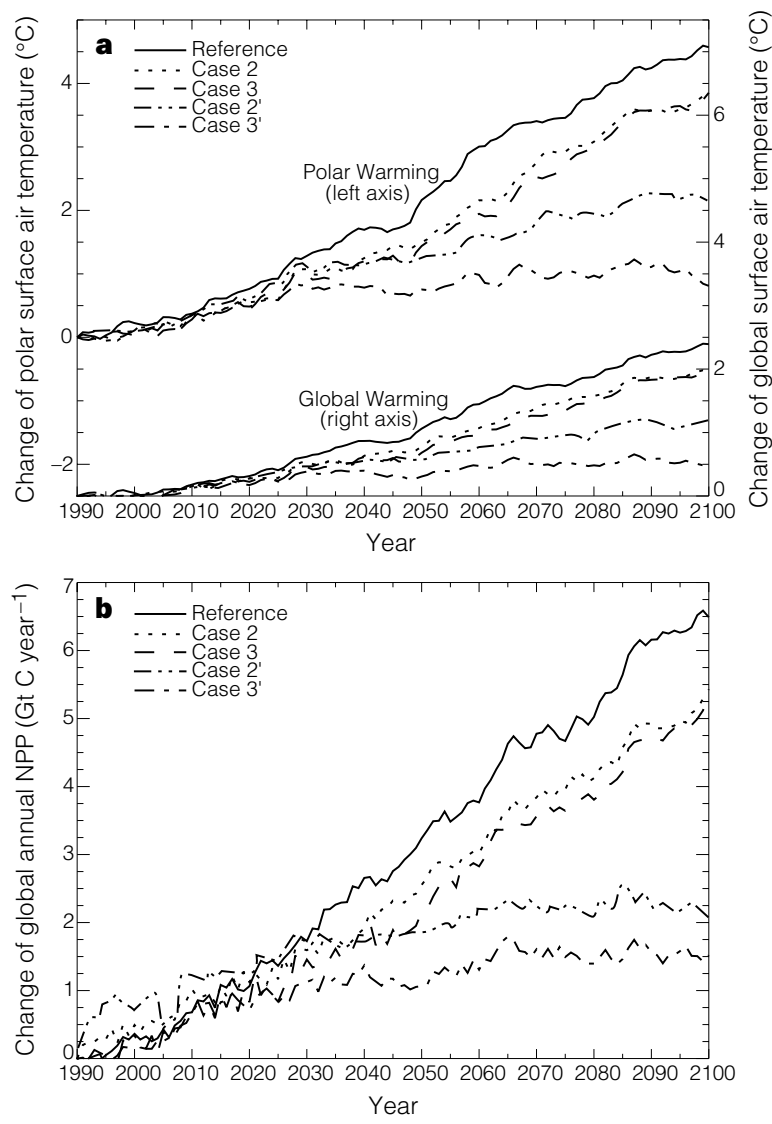

scenario. For other greenhouse gases, our marginal abatement curves are based on 'bottom-up' engineering studies that sometimes fail to include some economic costs and barriers to implementing reductions. In addition, measuring and monitoring sinks and emissions from very diverse sources of greenhouse-gas reductions could add to cost, or require a larger economic incentive than we have estimated, for emitters to undertake abatement measures. This set of concerns, many of which fall into the category of transaction costs, could mean that our marginal abatement curves underestimate costs.

Carbon dioxide emissions are also uncertain and depend on a number of assumptions and sensitivities, as has been shown elsewhere $^{12,37}$. The handling of technical change and costing of alternative technologies has generated widely varying estimates of

Figure 3 Climate and ecosystem effects. Panel a shows the three-year moving-average global and polar temperature changes from 1990 levels. With the Kyoto Protocol extended to 2100 (cases 2 and 3) there is a 17\% reduction in warming from the reference. With more aggressive policies after 2010 (cases $2^{\prime}$ and $3^{\prime}$ ) warming is reduced by about 55 and $85 \%$ respectively from the reference. Panel $\mathbf{b}$ shows the three-year moving average of net primary production changes from 1990 levels. With the Kyoto Protocol extended to 2100 (cases 2 and 3), the net primary production increases by $22 \%$ less than in the reference. With a more stringent policy (cases $2^{\prime}$ and $3^{\prime}$ ) the increase is about 65 and $77 \%$ less respectively. 
Case 1: $\mathrm{CO}_{2}$ target and control

Case 2: Multi-gas target, $\mathrm{CO}_{2}$ control

Case 3: Multi-gas target and control

\section{USA \\ 37.5
45.6}

27.8

\section{EEC \\ 30.0 \\ 44.2}

23.7
OOE

15.6

18.2

8.0

\section{EET}

8.9

11.4

9.2
JPN

34.0

42.2

30.8

Regional definitions are given in Table 1. Costs are measured as the integral from no reduction to the projected reduction level under our marginal abatement curves. Notionally, costs are the foregone

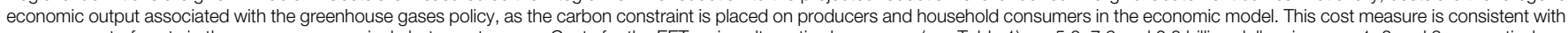
measurement of costs in the exogenous marginal abatement curves. Costs for the EET, using alternative base years (see Table 1) are 5.0, 7.9 and 6.0 billion dollars in cases 1, 2 and 3, respectively.

costs, some suggesting little or no cost for meeting the Kyoto $\operatorname{target}^{38}$. These costing approaches are not always complete or consistent with a fuller treatment of economic costs ${ }^{39}$. Recently attention has focused on the potential for a carbon constraint to induce technical change $e^{40}$, showing potentially large effects depending on the representation of the process. These differences can lead to cost differences or $\pm 50 \%$ in 2010 . Although the base cost estimate is uncertain, and marginal abatement curves themselves are uncertain, we would expect the basic conclusion, that there is substantial potential to reduce costs through inclusion of other gases, to hold. The comparison and ordering of case 1 versus case 3 is less certain and is more dependent on the varying growth of emissions of each gas and the sink potential. For example, delay beyond 2000 in starting a forestation programme could mean that very little sequestration is achieved by 2010 .

\section{Climate effects}

The new IGSM chemistry-radiation submodel used here ${ }^{13}$ yields a reference run with a predicted temperature increase from 1990 to 2100 of $2.4^{\circ} \mathrm{C}$. This is similar to the $2.6^{\circ} \mathrm{C}$ in previous reference runs ${ }^{12}$ despite several changes in EPPA and chemistry-radiation submodels. This is because the effects of the lower $\mathrm{CO}_{2}$ emissions predicted by EPPA are offset approximately by the emissions of HFCs, PFCs, and $\mathrm{SF}_{6}$ that were not included previously, whereas effects of the inclusion of the (predicted) increases in tropospheric ozone are offset approximately by higher $\mathrm{SO}_{x}$ emissions.

Predicted warming (and predicted sea-level rise) between 1990 and 2100 is lowered from the reference case by only $\sim 17 \%$ in cases 2 and 3 (Fig. 3a) and there is little difference between the cases. This small difference indicates that, for the Kyoto policy extended through 2100, the crude approximation represented by the GWPs does not give rise to substantial differences in temperature effects. The little difference that exists is difficult to distinguish from the natural variability simulated by the climate model; it illustrates the effects, in cases 2 and 3, of interactions (chemical and radiative) between the non- $\mathrm{CO}_{2}$ gases not captured by GWPs (such as the effects of added $\mathrm{CO}$ and $\mathrm{CH}_{4}$ on their principal sink ( $\mathrm{OH}$ radicals) and hence on the lifetimes of these two gases $\left.{ }^{41}\right)$. The absolute and percentage reduction in predicted global average warming is modest in cases 2 and 3, but the effect on absolute warming between 1990 and 2100 in the regions poleward of $51^{\circ} \mathrm{N}$ and $51^{\circ} \mathrm{S}$ is more substantial (Fig. 3a). Specifically, the average polar warming is lowered from about $4.6^{\circ} \mathrm{C}$ in the reference run to about $3.8^{\circ} \mathrm{C}$ in the case 2 and 3 runs, which may be important for the stability of terrestrial boreal and tundra ecosystems and of the Greenland and Antarctic ice sheets.

Cases $2^{\prime}$ and $3^{\prime}$, which impose much more substantial emissions cuts than in cases 2 and 3 , yield much more substantial reductions in predicted temperature increase compared with the reference. In addition, significant differences now occur between the two cases (Fig. 3a). In case $3^{\prime}$ the temperature increase is reduced by nearly $80 \%$ in 2100 and the global-average temperature temporarily stabilizes at $\sim 0.5^{\circ} \mathrm{C}$ above 1990 levels. At the same time $\mathrm{CO}_{2}$ levels are $\sim 480$ p.p.m. (parts per million) and are still rising. In contrast, for case $2^{\prime}$ the reduction is only $\sim 55 \%$ from the reference by 2100 , the temperature is about $1.1^{\circ} \mathrm{C}$ above 1990 , the $\mathrm{CO}_{2}$ levels are about 420 p.p.m., and the temperature is still rising. These differences reflect the fact that GWPs only provide a crude approximation of the effects of these gases on climate, and the errors that show up under our more stringent policy cases have important policy consequences. Use of current 100-year GWPs would mean that a multi-gas policy designed to stabilize the climate (such as that approximated by case $3^{\prime}$ ) may not, in fact, do so, if countries decide to meet the policy target using only reductions in carbon dioxide emissions and carbon sinks. The fact that case $3^{\prime}$ shows less warming than case $2^{\prime}$ means that other gases as a group are undervalued by 100 -year GWPs, compared with the predictions of our model which simulates the principal interactions among gases. If 100-year GWPs were perfect indices then the results from cases 2 and 3 would be identical; and the results from cases $2^{\prime}$ and $3^{\prime}$ would be identical.

The case of $\mathrm{CH}_{4}$ and its interactions with other gases illustrates deeper problems with GWPs. For example, as a result of the much higher case $2^{\prime}$ emissions (Fig. 2b) in 2100, the atmospheric levels of $\mathrm{CH}_{4}, \mathrm{CO}$ and ozone in case $2^{\prime}$ are respectively $2.8,1.8$ and 1.2 times those in case $3^{\prime}$. Through chemistry-climate interactions this leads to a $40 \%$ lower level in 2100 of the main atmospheric destroyer of $\mathrm{CH}_{4}$ (the hydroxyl free radical, $\mathrm{OH}$ ) and proportionately longer $\mathrm{CH}_{4}$ lifetimes (and, hence, a higher GWP), in case $2^{\prime}$ relative to case 3'. Global warming potentials reflect the cumulative forcing over the specified (for example, 100-year) horizon, rather than a radiative forcing effect that is relevant for a particular year. The emissions control path in case $3^{\prime}$, with gradually greater reductions in emissions through to 2100 , means that the majority of integrated $\mathrm{CH}_{4}$ emission reductions occur in the few decades approaching 2100. These GWP-weighted reductions in $\mathrm{CH}_{4}$ are largely reflected as less warming by 2100 because of its shorter lifetime. In contrast, the climate effects of the additional reductions in $\mathrm{CO}_{2}$ (in case $2^{\prime}$ relative to case $3^{\prime}$ ) that occur late in the next century, would be observed mostly over the succeeding century and beyond. In other words, different GWPs are needed for different magnitudes and relative proportions of $\mathrm{CO}_{2}$ and non- $\mathrm{CO}_{2}$ reductions and for different times for assessing climatic effects. These complexities defeat the utility of the index.

\section{Ecosystem response}

We report ecosystem effects in terms of net primary production (NPP), defined as the difference between plant carbon uptake by photosynthesis and loss by plant respiration, which is an important ecosystem variable. It reflects the ability of ecosystems to supply food and fibre and drives the rates of most "ecosystem services", including carbon storage ${ }^{42,43}$. The ability of terrestrial ecosystems to store anthropogenic carbon depends on the relative rates of NPP and the decomposition of organic matter. The five IGSM runs all show NPP increases, but all policy cases show smaller increases (Fig. 3b). Decomposition also increases in all scenarios, but at a slower rate than NPP, such that $161 \mathrm{GtC}$ is added to natural ecosystems between 1990 and 2100 in the reference case. In the model runs simulating the $\mathrm{CO}_{2}$-only approach (case 2) and the multi-gas approach (case 3), 127 and $130 \mathrm{GtC}$ respectively are added. Cases $2^{\prime}$ and $3^{\prime}$ show considerably smaller increases in NPP and greater differences occur between the cases. The NPP patterns for case $2^{\prime}$ and $3^{\prime}$ result from a complex set of interactions between plant and soil responses to changes in both $\mathrm{CO}_{2}$ concentration and nitrogen availability, as controlled by temperature 
changes. These results illustrate an additional limitation of GWPs. In particular, because the fertilization effect of rising $\mathrm{CO}_{2}$ increases NPP and carbon storage in ecosystems, there is a negative feedback on the climate system. GWPs do not capture this effect and thus the current GWP formulation undervalues all other gases in toto relative to $\mathrm{CO}_{2}$ (or, equivalently, overvalues $\mathrm{CO}_{2}$ relative to all other gases in toto).

The $\mathrm{CO}_{2}$ fertilization effect compensates for at least some of the climate-induced reductions in plant production and ecosystem storage, until the atmospheric $\mathrm{CO}_{2}$ concentration reaches a saturation level, after which there would be no additional beneficial effects. Both the magnitude of the effect and the saturation level are uncertain. Some ecological models, including ours, predict that the $\mathrm{CO}_{2}$ fertilization effect will be large enough to more than compensate for climate-induced reductions in plant production and ecosystem carbon storage throughout the twenty-first century. Other models predict that the climate-induced effects will dominate the ecosystem response and any $\mathrm{CO}_{2}$ fertilization compensation will disappear during the first half of the twenty-first century ${ }^{44}$.

Our analysis does not include the effects of the potential redistribution of ecosystem boundaries on terrestrial carbon dynamics or climate. Such redistribution shows up in extensive studies of species response to climate changes in the past. Both the speed of the redistribution and the areal extent of the dieback-reorganization sequence over a specific area are uncertain. One reason for this uncertainty is an incomplete understanding of the response of ecosystems to disturbances such as fires, high winds, ice storms, insects or other pathogens, particularly if there are large synchronous disturbances. In a modelling exercise that included large synchronous disturbances and species redistribution ${ }^{45}$, the estimated loss was as much as $200 \mathrm{GtC}$ before a new steady-state condition was established and net carbon storage occurred again in terrestrial ecosystems. This estimate probably represents an upper bound to the effect of biome shifts alone in causing a transient loss of carbon from the terrestrial biosphere. The faster the rate of climate change, the greater the likelihood of large transient carbon losses from terrestrial ecosystems due to biome shifts during the transient carbon losses ${ }^{46}$. Such losses would alter predicted $\mathrm{CO}_{2}$ levels and hence warming, compared to those in our study.

Other consequences of vegetation redistribution may be more subtle. Because boreal forests absorb more solar radiation than tundra does, predicted poleward shifts in the location of the foresttundra boundary during a period of warming can amplify climate changes by as much as $50 \%$ (ref. 47). The amplification of warming associated with the poleward movement of the boreal forest would alter the temperature changes implied in Fig. 3.

\section{Discussion}

Much current analysis and policy discussion narrows the climate issue to a debate about carbon emissions from fossil fuels. In contrast, we have analysed climate policy as negotiated under the Kyoto agreement including critical issues like forest sinks and the non- $\mathrm{CO}_{2}$ greenhouse gases. The analysis also explicitly considers atmospheric interactions among these gases, climate feedbacks, the role of $\mathrm{CO}$ and $\mathrm{NO}_{x}$, and the cooling effect of aerosols.

Economic analyses that leave out other trace gases err in several ways: reference emissions are understated, allowable emissions in the commitment period are too low, and opportunities to reduce emissions of other gases are not considered in abatement options. These effects are partially offsetting, so it is not possible to predict the direction of the error $a$ priori. Based on our modelling exercise we find that omitting non- $\mathrm{CO}_{2}$ gases and sinks leads to an overestimate of the annual cost in 2010 in Annex B regions overall, of $\sim 21 \%$. More important, however, is that achieving approximately the same reduction in warming by control of fossil $\mathrm{CO}_{2}$ only, ignoring other gases and sinks, could cost over $60 \%$ more. Failure to consider other trace gases and sinks also has differential regional effects that could affect the value of emissions trading. A striking effect in our estimates is that failure to reduce leakage from natural gas and coal mining operations could reduce 'hot air' in the FSU substantially. Further, the OOE becomes one of the lower-cost regions, whereas it is in fact high-cost if only carbon from fossil fuels is considered. Given the very few studies of the cost of control of non- $\mathrm{CO}_{2}$ gases, it is not possible to provide a quantitative estimate of probability ranges for these estimates. However, the potential from other gases appears to be substantial, perhaps greater than we have estimated; realizing this potential will require that policy incentives to control these other gases that minimize transactions costs be put in place.

The effects of using GWPs to express non- $\mathrm{CO}_{2}$ greenhouse-gas emissions in terms of equivalent amounts of $\mathrm{CO}_{2}$ on atmospheric composition, climate and ecosystems are relatively small if target reductions and participation in the agreement does not change from that negotiated under the Kyoto Protocol. But this is largely due to the relatively small role played by reductions in non- $\mathrm{CO}_{2}$ gases in this case. Under a much more stringent hypothetical policy that could approximately achieve stabilization of climate, large differences develop between a multi-gas and a $\mathrm{CO}_{2}$-only strategy. This exposes a significant weakness in the GWP approach. In fact, our predictions show that using Kyoto-specified GWPs means that the choice of control strategy could affect whether or not stabilization of radiative forcing is achieved. These differences develop early in the next century, soon after the point where we suggest that the agreement is extended to developing countries where emissions of (and hence potential reductions in) non- $\mathrm{CO}_{2}$ greenhouse gases are more substantial.

Throughout we have tried to identify significant uncertainties in our analysis. Our reference scenario should be viewed as one plausible picture of the future rather than a most-likely prediction. The Kyoto policy, although it has been signed, still awaits ratification by the necessary number of countries. Our suggested extensions of policies beyond 2010 were designed to illustrate climate and ecosystem effects, and are not intended to represent economically acceptable, fair, or politically feasible extensions of the Kyoto Protocol. Here we have examined the effects of the addition of other gases and sinks in climate-change policy; we find that these effects could be significant. More research is required in order to understand the role of these gases in mitigating potential climate change.

\section{Received 14 January; accepted 18 August 1999}

1. Adams, R. M., Chang, C., McCarl, B. \& Callaway, J. in Global Change: Economic Issues in Agriculture, Forestry, and Natural Resources (eds Reilly, J. \& Anderson, M.) 273-287 (Westview, Boulder, CO, 1992).

2. Richards, K. R. in Global Change: Economic Issues in Agriculture, Forestry, and Natural Resources (eds Reilly, J. \& Anderson, M.) 288-310 (Westview, Boulder, CO, 1992).

3. Cook, E. Lifetime Commitments: Why Policy-makers Can't Afford to Overlook Fully Fluorinated Compounds (World Resources Institute, Washington DC, 1995).

4. Harnisch, J. \& Prinn, R. G. Sulfur hexafluoride emissions. Environ. Sci. Technol. 4, 56a (1999).

5. Harnisch, J., Sue Wing, I., Jacoby, H. D. \& Prinn, R. G. in Extraction and Processing Division Congr. 1999 (ed. Mishra, B.) 797-815 (The Minerals, Metals and Materials Soc., Warrendale, PA, 1999).

6. Victor, D. G. \& MacDonald, G. J. Future Emissions of Sulfur Hexafluoride and Perfluorocarbons: Implications for Global Policy and Verifying Compliance with the Kyoto Protocol (International Institute for Applied Systems Analysis, Laxenburg, 1998).

7. Victor, D. G. \& MacDonald, G. J. A model for estimating future emissions of sulfur hexafluoride and perfluorocarbons. Clim. Change (in the press).

8. Hoffert, M. I. et al. Energy implications of future stabilization of atmospheric $\mathrm{CO}_{2}$ content. Nature 395, 881-884 (1998).

9. Wigley, T. M., Richels, R. \& Edmonds, J. A. Economic and environmental choices in the stabilization of climate. Nature 379, 240-243 (1996)

10. Nordhaus, W. D. Managing the Global Commons 49-74 (MIT Press, Cambridge, MA, 1994).

11. Hourcade, J. C. et al. in Climate Change 1995: Economic and Social Dimensions of Climate Change (eds Bruce, J. P. et al.) 297-366 (Cambridge Univ. Press, 1996).

12. Prinn, R. et al. Integrated global system model for climate policy assessment: feedbacks and sensitivity studies. Clim. Change 41, 469-546 (1999).

13. Reilly, J.et al. Multi-Gas Assessment of the Kyoto Protocol (MIT Joint Program on the Science and Policy of Global Change, Report No. 45, MIT, Cambridge, MA, 1999).

14. Yang, Z. et al. The MIT Emissions Prediction and Policy Analysis (EPPA) Model (MIT Joint Program on the Science and Policy of Global Change, Report No. 6, MIT, Cambridge, MA, 1996). 
15. Wang, C., Prinn, R. G. \& Sokolov, A. A global interactive chemistry climate model: formulation and testing. J. Geophys. Res. 103, 3399-3417 (1998).

16. Sokolov, A. P. \& Stone, P. H. A flexible climate model for use in integrated assessment. Clim. Dyn. 14, 291-303 (1998)

17. Xiao, X. et al. Transient climate change and net ecosystem production of the terrestrial biosphere. Glob. Biogeochem. Cycles 12, 345-360 (1998).

18. Schimel, D. et al. in Climate Change 1995: the Science of Climate Change (eds Houghton, J. T. et al.) 65131 (Cambridge Univ. Press, 1996).

19. Fan, S. et al. A large terrestrial carbon sink in North America implied by atmospheric and oceanic carbon dioxide data and models. Science 282, 442-446 (1998).

20. Schneider, S. H. Detecting climatic change signals: are there any fingerprints? Science 263, 341-347 (1994).

21. Eckaus, R. Comparing the effects of greenhouse gas emissions on global warming. Energy J. 13, 25-34 (1992).

22. Reilly, J. \& Richards, K. Climate change damage and the trace gas index issue. Environ. Resour. Econ. 3 41-61 (1993).

23. Schmalensee, R. Comparing greenhouse gases for policy purposes. Energy J. 14, 245-255 (1993).

24. Reilly, J. in Economics and Policy Issues in Climate Change (ed. Nordhaus, W. D.) 243-256 (Resources for the Future, Washington DC, 1998).

25. Lind, R. \& Schuler, R. in Economics and Policy Issues in Climate Change (ed. Nordhaus, W. D.) 59-96 (Resources for the Future, Washington DC, 1998).

26. Energy Information Administration (EIA) International Energy Outlook (Rep. No. DOE/EIA 0484(99)); 〈http://www.eia.doe.gov/oiaf/ieo99).

27. Nilsson, S. \& Schopfhauser, W. The carbon-sequestration potential of a global afforestation program. Clim. Change 30, 267-293 (1994).

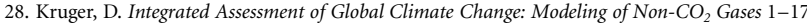
(Methane and Utilities Branch, US Environmental Protection Agency, Washington DC, 1999).

29. US Environmental Protection Agency, Methane Branch. Costs of Reducing Methane Emissions in the United States 1-92 (1999); http://www.epa.gov/ghginfo

30. Cole, V. et al. in Climate Change 1995: Impacts, Adaptations, and Mitigation of Climate Change (eds Watson, R. et al.) 726-771 (Cambridge Univ. Press, 1996).

31. Denbaly, M. \& Vroomen, H. Dynamic fertilizer nutrient demands for corn: a cointegrated and errorcorrection system. Am. J. Agric. Econ. 75 (1) (1993).

32. Fernandez-Cornejo, J. Demand and Substitution of Agricultural Inputs in the Central Cornbelt States (Rep. TB-1816, US Dept of Agriculture, Economic Research Service, Washington, 1993).

33. Oram, D. E., Sturges, W. T., Penkett, S. A., McCulloch, A. \& Fraser, P. J. Growth of fluoroform $\left(\mathrm{CHF}_{3}\right.$ HFC-23) in the background atmosphere. Geophys. Res. Lett. 25, 35-38 (1998).

34. McCulloch, A. Future consumption and emissions of hydrofluorocarbon (HFC) alternatives to CFCs: comparison of estimates using top-down and bottom-up approaches. Environ. Int. 21, 353-362 (1995)

35. Maiss, M. \& Brenninkmeijer, C. A. M. Atmospheric $\mathrm{SF}_{6}$, trends, sources and prospects. Environ. Sci. Technol. 32, 3077-3086 (1998)

36. de Jager, D., Hendriks, C. A., Heijnes, H. A. M. \& Blok, K. in Greenhouse Gas Control Technologies (eds Eliasson, B., Riemer, P. W. F. \& Wokaun, A.) 503-508 (Pergamon, New York, 1999).

37. Jacoby, H. et al. $\mathrm{CO}_{2}$ limits: economic adjustments and the distribution of burdens. Energy J. 18 (3), 31-58 (1997).

38. US DOE Interlaboratory Working Group. Scenarios of US Carbon Reductions (Office of Efficiency and Renewable Energy, US Dept of Energy, Washington DC, 1997).

39. Jacoby, $\mathrm{H}$. The uses and misuses of technology development as a component of climate policy 1-16 (MIT Joint Program on the Science and Policy of Global Change, Report No. 43, MIT, Cambridge, MA, 1998).

40. Goulder, L. \& Schneider, S. Induced technical change and the attractiveness of $\mathrm{CO}_{2}$ abatement policies. Resour. Energy Econ. 21, 211-253 (1999).

41. Wang, C. \& Prinn, R. G. Impact of emissions, chemistry, and climate on atmospheric carbon monoxide: 100-year predictions from a global chemistry-climate model. Chemosphere (in the press); (also as the MIT Joint Program on the Science and Policy of Global Change Report No. 35, MIT, Cambridge, MA, 1998).

42. Daily, G. C. (ed.) Daily Services: Societal Dependence on Natural Ecosystems (Island Press, Washington DC, 1997).

43. Melillo, J. M. Warm, warm on the range. Science 283, 183-184 (1999).

44. Cao, M. \& Woodward, F. I. Dynamic responses of terrestrial ecosystem carbon cycling to global climate change. Nature 393, 249-252 (1998).

45. Smith, T. M. \& Shugart, H. H. The transient response of terrestrial carbon storage to a perturbed climate. Nature 361, 523-526 (1993).

46. Woodwell, G. M. et al. in Biotic Feedbacks in the Global Climatic System (eds Woodwell, G. M. \& Mackenzie, F. T.) 393-411 (Oxford Univ. Press, NY, 1995).

47. Foley, J. A. et al. Feedbacks between climate and boreal forests during the mid-Holocene. Nature 371, 52-54 (1994).

\section{Acknowledgements}

We acknowledge the financial support of the industrial and government sponsors of the Joint Program on the Science and Policy of Global Change at MIT, and thank V. Webb for his research assistance.

Correspondence should be addressed to J.R. (e-mail: jreilly@mit.edu). 